\title{
Multidisciplinary Joint Clinics: Talent Wins Games, but Teamwork Wins Championships
}

\begin{abstract}
Cancer is among the leading causes of deaths worldwide. The treatments of cancer across most sites involve using surgery, systemic therapy, and radiation therapy. The treatment protocols are complex and require careful planning at the beginning of the therapy and coordination between the treating teams, the patient, and the caregivers to ensure compliance and avoid unnecessary treatment delays. This commentary provides an insight into the role the multidisciplinary joint clinics play in providing personalized cancer care. While such joint clinics are advantageous, they are not devoid of drawbacks and these are also enumerated. In this era, when communication platforms are increasingly digitized, we have highlighted the need for virtual tumor boards. The commentary aims to motivate the development of multidisciplinary joint clinics for ensuring holistic cancer care across the country.
\end{abstract}

Keywords: Holistic care, joint clinics, multidisciplinary care, personalized medicine, virtual tumor board

\section{Introduction}

According to the Globocan 2018 report, cancer is the second leading cause of death worldwide. ${ }^{[1]}$ The treatment protocols for different types of cancers become standard of care based on long-term results from prospective studies conducted on thousands of patients and are followed by most oncologists across the globe. These protocols are updated regularly on the basis of advances in the fields and evidence from newer studies. Despite these advances and complex therapies, the differences in the cancer-specific and overall survival among patients across different stages of cancer have remained significant and indicate a need for further improvement. ${ }^{[2-4]}$ The NCCN guidelines are a good example of the already complex web of cancer treatments and how each treatment modality is sequenced and integrated for improving the outcomes for these patients. ${ }^{[5]}$ It emphasizes the need for coordination and understanding among different physicians, health-care workers, patients, and caregivers. In the real-world setting, providing information on the different aspects of cancer, its treatment, acute and late toxicities and variable outcomes can be challenging.

This is an open access journal, and articles are distributed under the terms of the Creative Commons Attribution-NonCommercial-ShareAlike 4.0 License, which allows others to remix, tweak, and build upon the work non-commercially, as long as appropriate credit is given and the new creations are licensed under the identical terms.

For reprints contact: WKHLRPMedknow reprints@wolterskluwer.com

\section{Increasing Complexities in Cancer Care Decision Pathways}

Improvement in education levels in our country along with digitalization and improved access to the web-based information has contributed to improved cancer awareness. The Internet has also brought about a change in the way patients access information empowering them to take well-informed treatment decisions. This is constantly challenging the archaic way of clinician's undemocratic approach toward patients' treatment choices. The focus is now shifting from a disease-directed approach to a patient-centric approach. ${ }^{[6]}$ This requires the oncologists to address several new domains of a patient's welfare like the psychosocial and mental well-being, genetic testing for somatic and germ line mutations, along with issues of survivorship such as fertility, endocrine imbalances, menopause, and quality of life among others. Individually meeting all the physicians prior to starting therapy to chalk out a comprehensive care plan and discussing various aspects of cancer care can lead to significant delays in instituting therapy and can negatively impact the outcome of the patients. Therefore, a meeting with the multidisciplinary team together in a clinic can save time and

\footnotetext{
How to cite this article: Pathak RS, Shylasree TS, Bajpai J. Multidisciplinary joint clinics: Talent wins games, but teamwork wins championships. Indian J Med Paediatr Oncol 2020;41:804-8.
}

\section{Rima Sanjay \\ Pathak', TS Shylasree ${ }^{2}$, Jyoti Bajpai ${ }^{3}$}

${ }^{1}$ Department of Radiation Oncology, Tata Memorial Centre, Homi Bhabha National Institute, Mumbai, Maharashtra, India, ${ }^{2}$ Department of Gynecological Oncology, Tata Memorial Centre, Homi Bhabha National Institute, Mumbai, Maharashtra, India, ${ }^{3}$ Department of Medical Oncology, Tata Memorial Centre, Homi Bhabha National Institute, Mumbai, Maharashtra, India

Submitted: $27-A p r-2020$

Revised: 12-May-2020

Accepted: 30-Jun-2020

Published: 31-Dec-2020

Address for correspondence: Dr. Jyoti Bajpai, Department of Medical Oncology, Room 1115, $11^{\text {th }}$ Floor, Homi Bhabha Block, Tata Memorial Centre, Parel, Mumbai - 400 012, Maharashtra, India.

E-mail:dr_jyotibajpai@yahoo. co.in

Access this article online Website: www.ijmpo.org

DOI: 10.4103/ijmpo.ijmpo_203_20 Quick Response Code:

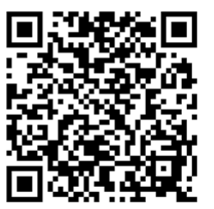


resources and provide additional advantages that are listed in Figure 1a-c.

\section{Key Players of the Multidisciplinary Joint Clinics}

Multidisciplinary joint clinics are clinics where people from multiple specialties gather at a pre-specified time to discuss the approach or course of treatment for patients. For example, it is mandatory that at least one person from each of the three main modalities such as the surgical oncologist, medical oncologist, and radiation oncologist be present for breast cancer joint clinics. While it is desirable to have a long list of team members [Figure 2], the clinic may invite members included in the acceptable/desirable category as and when required. The list of team members that are needed for discussion may vary with the type of cases to be discussed including the sites of cancer and the lists in Figure 2 are provided just as an example. While there are advantages to a multidisciplinary joint clinic [Figure 1a-c], setting the clinic up and getting it to function smoothly are quite challenging.

\section{Challenges of Establishing Joint Clinics in Low- and Middle-Income Countries}

In low- and middle-income countries with skewed doctorpatient ratio, especially in the oncology sector, one of the biggest challenges is finding time for a joint clinic in an already busy schedule. ${ }^{[7,8]}$ The other major challenge is to convince the hospital administration to support the joint clinics by providing space, infrastructure, and logistic support. In addition, the hospital should also provide remuneration for the time spent in the joint clinics so that the oncologists participate without any hesitation. ${ }^{[9-11]}$ In the government/charitable hospitals where the paycheck of the treating oncologist may not depend on the total number of patients treated/income generated by the oncologist, there may be less resistance to starting such clinics. On the contrary, in the corporate hospital set-up, infrastructure and space for the clinic may be relatively easier to get but

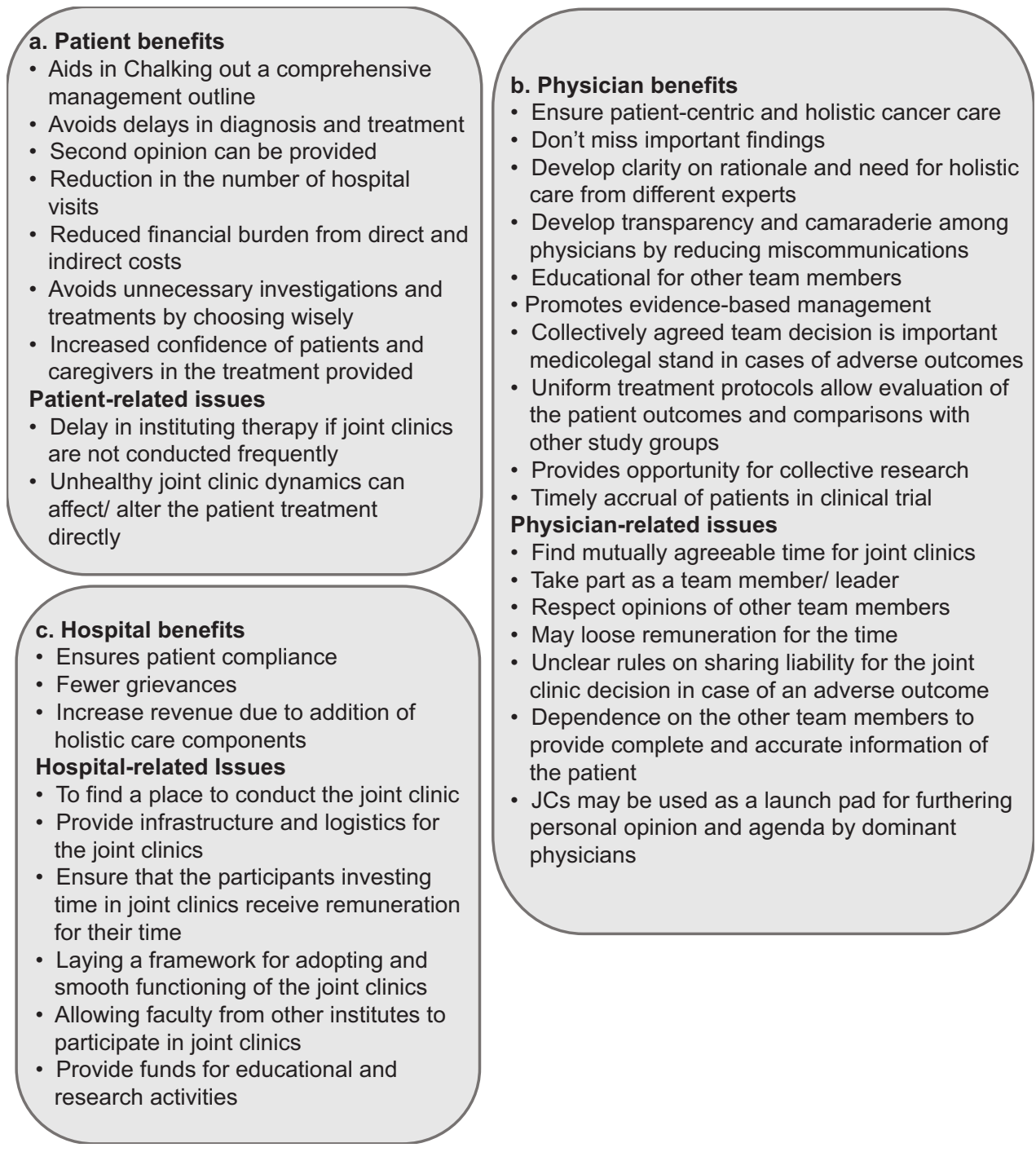

Figure 1: (a-c) Benefits and challenges of multdisciplinary joint clinics for the patient, physician, and the hospital 


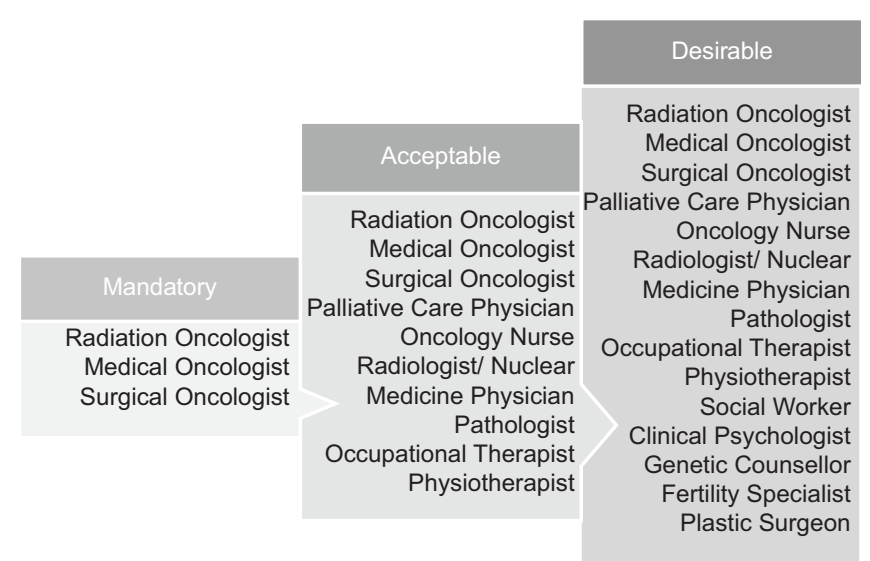

Figure 2: List of team members known to impact patient's management that should/may participate in multidisciplinary joint clinics, grouped into mandatory, acceptable, and desirable categories

the hospital administration may not favor setting-up joint clinics as they do not directly generate income for the hospital. ${ }^{[7]}$ The other challenge specific to smaller treatment centers is the unavailability of all the specialties required for the joint clinic. This can be overcome by making provisions for inviting members from other institutes from the same region which also generates a robust patient referral mechanism and potentially improves coordination between the treating physicians during sequencing of therapies or institution of concurrent therapies.

\section{Framework to Establish Multidisciplinary Joint Clinics}

The government policy of "health for all" has emphasized on bio-psycho-socio-economic dimensions of health and illness. The competency-based undergraduate curriculum by Medical Council of India also aspires to produce clinicians competent to provide holistic care and lead multi-disciplinary teams in future in alignment with the government policies. ${ }^{[7]}$ Accreditation agencies for hospitals and various aspects of health care are strong proponents of teamwork and lay great emphasis on coordination processes within the health-care framework. Acquiring accreditation from such agencies improves patient trust on the quality of care and provides additional value to the hospital. ${ }^{[12,13]}$ When starting a joint clinic at an institute, a detailed framework distributing the roles and responsibilities of the individuals participating should be laid down. This provides clarity to the individuals and improves participation. The members of the clinic have the responsibility to respect the decisions taken in the clinic. These decisions are expected to be evidence based and ethically sound. It is possible to sustain this effort only if everyone respects others' opinions and time by conducting the meeting and arriving for the meeting on time. It is advisable to discuss all the cases in a multidisciplinary manner and ideally circulated among the team members well in advance for a fruitful discussion, especially when dealing with unusual cases or circumstances. ${ }^{[14]}$

\section{Legal Aspects of Multidisciplinary Joint Clinics} and Their Decisions

Multidisciplinary joint clinics are not a registered body, and hence, they do not have a legal standing as an entity. Nevertheless, all the members of the team share a collective responsibility toward the decisions made. ${ }^{[14]}$ Since these joint clinics are meant to be horizontal meetings devoid of hierarchy, each individual shares the same level of responsibility for the decisions taken during the meeting. However, the court of law may not hold individuals responsible for the decisions taken in a joint meeting which were beyond their scope of expertise. ${ }^{[14]}$ The decision-making process can be variable across such clinics where some go by majority, whereas others consider unanimous vote. Most of the time, a majority vote is accepted. There may be individuals in the meeting who do not agree with the decision taken by the clinic yet share the collective responsibility of the clinic decision. ${ }^{[10]}$ In case of an adverse outcome of such a decision, the individual in disagreement with the clinic decision would be found equally responsible for the outcome unless his or her disagreement is clearly documented in the proceedings or minutes of the meeting. This highlights the importance of clarity and detailed documentation required during the joint clinics. Recall of the events during the joint clinic several months later may not be possible, and hence, the documentation should be done preferably on the same day as the clinic.

\section{Drawbacks of Multidisciplinary Joint Clinics}

Multidisciplinary clinics are not devoid of any drawbacks. Most of them are listed in Figure 1a-c. These too need to be highlighted and strategies should be devised to prevent them from occurring. Frequently, in hospitals with hierarchy-based clinics, these joint clinics soon lose the horizontal structure and senior physicians dominate. Such a team may not be able to uphold the best interest of the patient and goes against the ethos of a multidisciplinary team. ${ }^{[10]}$ On rare occasions, when a consensus cannot be reached, the patient's treatments can be delayed. For a busy institute, it may not be possible to discuss all patients in a multidisciplinary team, and standard selection criteria may be implemented for discussing them in joint clinics. ${ }^{[10]}$ Meetings where patients are not evaluated directly suffer from the lack of patient representation and do not provide an opportunity to choose between the options if there are any. These drawbacks can be corrected if there is a clinical decision aid which is customized to the needs of the hospital or setup that can guide to make decision making uniform, consistent, and evidence-based. Even with these inconsistencies, such joint clinics have shown to improve the quality of cancer care and therefore have been adopted by most institutes. 


\section{Virtual Tumor Boards}

While multispecialty hospitals in metropolitan cities or tier II cities may have all the required specialties working together in the same hospital, this may not be the case in more remote areas or smaller hospitals and cancer clinics. This may make it more difficult to organize a multidisciplinary joint clinic. However, patients can still be given access to joint clinics using video conferencing and such meetings are called virtual tumor boards (VTBs). VTBs allow all the specialists to participate in the meeting without physically being present at the clinic. VTBs are gaining popularity in India because of its potential to seamlessly integrate within existing hospital space. Since Internet has no boundaries, specialists from across different parts of the countries and different countries can participate in the tumor boards and provide an expert opinion. The National Cancer Grid VTBs are such a need-based solution that connect different parts of the country where all the members of the grid can attend the tumor board by logging in from across the globe provided that they are connected to the Internet. ${ }^{[15]}$ The advantages of such VTBs in a country like India are manifold. Other than the advantages already mentioned in Figure 1a-c, VTBs are cost-effective where they save travel time for the patient and the specialist and do not require hospital or clinic space for a large gathering. It, of course, requires an initial investment in providing audio-visual and Internet support at the hospital that hosts the VTB. However, cost is negligible compared to the invaluable opinions the patients get in return from a multidisciplinary approach. Furthermore, considering that such a multidisciplinary approach is considered a standard for cancer care, non-availability of multiple specialists to make a joint decision at a smaller cancer center may open themselves up for a potential lawsuit in cases of adverse clinical outcomes. ${ }^{[14-17]}$

There are limited tertiary or regional cancer centers in the country. ${ }^{[18]}$ It becomes difficult for an oncologist to refer the patients for further care to these centers due to the social and financial constraints of the patients. VTB discussions can form a perfect solution where the treating oncologist can learn from the experiences and cases from across the country and train in providing interspecialty care under special circumstances. It also helps centers from tier 2 and 3 cities to coordinate referral, diagnostics, and follow-ups with the tertiary care hospitals and vice versa. Common discussion platforms like these help all the centers across the country to develop and adopt a uniform model of patient care for both common and uncommon cancers reducing the disparities in cancer care in different parts of the country. Such opportunities transform into great platforms for multilateral teaching and learning. ${ }^{[1,16,19]}$ Over a period of time, one can recognize patterns of cases and issues discussed in the VTBs, highlighting challenges in cancer care, including resource, skill, and health personnel constraints, across different parts of the country.
Recurring themes and decision algorithms can be stored as sample cases or learning modules to prevent repetition of similar cases. A similar online opinion platform, "TMC NCG-Navya," was able to build an artificial intelligence model based on the expert opinions provided which could accurately predict the tumor board opinions more than $90 \%$ of the time. ${ }^{[20]}$ These models once validated can then be used at smaller or remote cancer centers for immediate assistance, making them more time and cost-efficient. ${ }^{[21]}$

The consensus opinion of a VTB, similar to the multidisciplinary joint clinic, is considered as an expert opinion which provides additional supports to the treating physician and is medicolegally binding for all those who participated in the VTB. Therefore, the patient should be made aware of the differences of opinion brought forward in the VTB before making an informed decision. While VTB is a great platform, it is not devoid of any limitations. The drawbacks specific to VTBs are related to the dysfunctional technology, concerns about confidentiality, issues pertaining to sharing confidential patient data, issues with a breach in cybersecurity, coordination challenges between hospitals, and limited patient centeredness among others. ${ }^{[11,19,22]}$ Nevertheless, the advantages far outweigh the drawbacks, and hence, multidisciplinary cancer patient care must be implemented whether through physical meetings, VTBs, or a hybrid model where people can participate either physically or virtually depending on their comfort. This approach also helps to share the liability through collective responsibility. Even when treating common cancers where individuals feel confident in instituting protocol-based treatment, the multidisciplinary discussion always adds a different perspective providing for holistic care. Therefore, individually we may win matches, but collectively we can win championships.

\section{Acknowledgments}

All the patients, oncologists, and other specialties who have contributed toward developing, continuing, and propagating multidisciplinary care even with busy schedules at the Tata Memorial Centre.

\section{Financial support and sponsorship}

Nil.

\section{Conflicts of interest}

There are no conflicts of interest.

\section{References}

1. World Health Organization. Globocan 2018: All Cancers. Int Agency Res Cancer 2019;1:1-2.

2. Breast Cancer Survival Statistics Cancer Research UK. Available from: https://www.cancerresearchuk.org/health-professional/ cancer-statistics/statistics-by-cancer-type/breast-cancer/ survival\#heading-Three. [Last accessed on 2020 Jan 20].

3. Agarwal G, Ramakant P. Breast cancer care in India: The current 
scenario and the challenges for the future. Breast Care (Basel) 2008;3:21-7.

4. Nair N, Shet T, Parmar V, Havaldar R, Gupta S, Budrukkar A, et al. Breast cancer in a tertiary cancer center in India - An audit, with outcome analysis. Indian J Cancer 2018;55:16-22.

5. National Comprehensive Cancer Network. NCCN Clinical Practice Guidelines in Oncology. Available from: https://www. nccn.org/professionals/physician_gls/default.aspx\#site. [Last accessed on 2020 April 27].

6. Tinetti ME, Naik AD, Dodson JA. Moving from disease-centered to patient goals-directed care for patients with multiple chronic conditions: Patient value-based care. JAMA Cardiol 2016;1:9-10.

7. Bajpai V. The Challenges Confronting Public Hospitals in India, Their Origins, and Possible Solutions. Adv Public Heal 2014;2014:1-27.

8. Blackwood O, Deb R. Multidisciplinary team approach in breast cancer care: Benefits and challenges. Indian J Pathol Microbiol 2020;63:S105-S112.

9. Meguid C, Ryan CE, Edil BH, Schulick RD, Gajdos C, Boniface $\mathrm{M}$, et al. Establishing a framework for building multidisciplinary programs. J Multidiscip Healthc 2015;8:519-26.

10. Gupta T. Multidisciplinary clinics in oncology: The hidden pitfalls. J Oncol Pract. 2007;3:72-3.

11. Rosell L, Wihl J, Nilbert M, Malmström M. Health professionals' views on key enabling factors and barriers of national multidisciplinary team meetings in cancer care: A qualitative study. J Multidiscip Healthc 2020;13:179-86.

12. O'Daniel M, Rosenstein AH. Professional communication and team collaboration. Patient Saf Qual An Evidence-Based Handb Nurs 2008;2:271-84.

13. Busse R, Klazinga N, Panteli D, Quentin W. Health policy series No. 53 The Editors Improving Healthcare Quality in Europe Characteristics, Effectiveness and Implementation of Different Strategies. Denmark; 2019. http://www.healthobservat ory.eu. [Last accessed on 2020 Apr 26].

14. Mental Health Commission. Multidisciplinary Team Working: From Theory to Practice; 2006. Available from: https://www.
mhcirl.ie/File/discusspapmultiteam.pdf. [Last accessed on 2020 April 25].

15. National Cancer Grid. Virtual Tumour Board NCG. Available from: https://tmc.gov.in/ncg/index.php/ activities-ncgvrtual-tumor-board. [Last accessed on $2020 \mathrm{Apr}$ 26].

16. Aston SJ, Reade S, Petersen B, Ward C, Duffy A, Nsutebu E. Extraordinary virtual multidisciplinary team meetings - A novel forum for the coordinated care of patients with complex conditions within a secondary care setting. Futur Healthc J 2018;5:218-23.

17. Shea CM, Teal R, Haynes-Maslow L, McIntyre M, Weiner BJ, Wheeler SB, et al. Assessing the feasibility of a virtual tumor board program: A case study. J Healthc Manag 2014;59:177-93.

18. India Against Cancer. Most Important Cancer Treatment Centers in India. Available from: http://cancerindia.org.in/ cancer-treatment-centers/. [Last accessed on 2020 Mar 02; Last accessed on 2020 Apr 26].

19. Hammer RD, Prime MS. A clinician's perspective on codeveloping and co-implementing a digital tumor board solution [published online ahead of print, 2020 Jan 23]. Health Informatics J 2020;1460458219899841.

20. Nair N, Gupta S, Ramarajan N, Srivastava G, Parmar V, Munshi A, et al. Validation of a software based clinical decision support system for breast cancer treatment in a tertiary care cancer center in India [abstract]. In: Proceedings of the ThirtySeventh Annual CTRC-AACR San Antonio Breast Cancer Symposium: 2014 Dec 9-13; San Antonio, TX. Philadelphia (PA): AACR; Cancer Res 2015;75(9 Suppl):Abstract nr P4-16-01.

21. Vora T, Khanna N, Prasad M, Ramarajan N, Srivastava G, Bhat V, Qureshi S, et al. Prospective comparison of travel burden, cost and time to obtain pediatric multidisciplinary tumor board treatment plan through in-person visits vs. an AI enabled health technology. Pediatr Blood Cancer 2019;66:e27989.

22. Acai A, Sonnadara RR, O’Neill TA. Getting with the times: A narrative review of the literature on group decision making in virtual environments and implications for promotions committees. Perspect Med Educ 2018;7:147-55. 